\title{
Magnetic resonance black-blood imaging to facilitate diagnosis of cerebral venous thrombosis?
}

\author{
Simon Fandler-Höfler ${ }^{1} \mathbb{D} \cdot$ Thomas Gattringer $^{1,2}$
}

Received: 19 October 2021 / Revised: 19 October 2021 / Accepted: 28 October 2021 / Published online: 21 January 2022

(c) The Author(s), under exclusive licence to European Society of Radiology 2022

Cerebral venous thrombosis (CVT) is a rare but important type of stroke, most frequently affecting young female adults aged 20-50 [1]. The incidence of CVT has risen over the last decades, partly due to shifts in prothrombotic risk factors, but mainly explained by an increased disease awareness and availability as well as improvement of noninvasive neuroimaging techniques leading to an increased rate of diagnosis [1]. Depending on the location, extension, and prevailing pathophysiology of CVT, presenting symptoms may vary widely from isolated headache to seizures, focal neurological deficits, and disorders of mental status. Heterogeneity of clinical presentation with atypical symptoms and challenges in radiological diagnostics explain why diagnosis of CVT can be notoriously difficult in clinical practice, illustrated by a median time of symptom onset to diagnosis of 7 days in a large multicenter study [2].

Widely available non-contrast CT has only a moderate sensitivity (but high specificity) for CVT [3]. CT venography has been shown to have an excellent sensitivity and specificity for CVT, although it may have limited value in diagnosis of cortical vein thrombosis [4]. Numerous MRI techniques are available to visualize cerebral venous structures with various predictive values reported, including fluid-attenuated inversion recovery, susceptibility-weighted imaging, non-contrast MRI venography such as time-offlight venography and contrast-enhanced MRI venography. Each has individual limitations, but the combination of multiple MRI sequences leads to excellent sensitivity and

This comment refers to the article available at https://doi.org/ 10.1007/s00330-021-08286-x.

Simon Fandler-Höfler

simon.fandler@medunigraz.at

1 Department of Neurology, Medical University of Graz, Auenbruggerplatz 22, 8036 Graz, Austria

2 Division of Neuroradiology, Vascular and Interventional Radiology, Department of Radiology, Medical University of Graz, Graz, Austria specificity for CVT. Advantages of MRI include clearer visualization of parenchymal lesions and no usage of ionizing radiation, while CT venography is often easier available; their sensitivity and specificity for CVT have been found to be comparable, although results from individual studies vary widely [5].

Quite recently, magnetic resonance black-blood thrombus imaging has been suggested to additionally aid in diagnosis by showing excellent sensitivity for CVT in two smaller studies. [6, 7] Both studies used a T1 3D variable-flip-angle turbo spin echo (SPACE) sequence on 3-T MRI scanners. In this issue of European Radiology, Yang et al extend on their initial study and report findings from a larger study cohort [8]. In their current study, they retrospectively investigated two groups of patients with suspected CVT: 114 patients with available magnetic resonance black-blood thrombus imaging and 97 patients without availability of this sequence. The authors report that neuroimaging which included magnetic resonance black-blood thrombus imaging had a higher rate of correct diagnosis of CVT with $94.7 \%$ (as defined by a multidisciplinary conference) compared to other neuroimaging techniques.

Additionally, the authors report that black-blood thrombus imaging allowed for accurate staging of thrombosed segments (into acute, subacute, and chronic groups, defined by the time from symptom onset to imaging and D-dimer levels). However, this conclusion needs to be interpreted with caution as serum D-dimer levels are not an established method to rate the stage of a cerebral thrombus and retrospective studies have known limitations regarding the exact assessment of patients' medical history including symptom onset. Differentiating an acute, subacute, and chronic thrombus would have been possible with more confidence by rating its imaging appearance on different conventional MRI sequences (T1, T2/FLAIR, SWI, or GRE T2*, depending on the oxygenation state of hemoglobin).

This study adds to the previous preliminary reports of magnetic resonance black-blood thrombus imaging for 
diagnosis of CVT. However, a number of limitations need to be considered. This was a retrospective study from a single specialized center, well-experienced in CVT imaging in general and black-blood thrombus imaging in particular (as the first study on magnetic resonance black-blood thrombus imaging for CVT was also performed at this center). The study cohort was likely specific to that setting and the majority of patients had subacute or chronic CVT. Therefore, results may not be easily translated to other centers, as patient populations, CVT acuity, and experience with differing MRI protocols may vary. Furthermore, acquisition of magnetic resonance black-blood thrombus sequences takes a significant amount of time, which may be a relevant limitation in a clinical setting.

Nonetheless, magnetic resonance black-blood thrombus imaging appears to be a welcome addition to the toolset of neuroimaging techniques for diagnosis of CVT. Larger and multicenter studies, including in centers previously naïve to black-blood thrombus imaging, are required to show whether it will find a place in daily clinical use.

Funding The authors state that this work has not received any funding.

\section{Declarations}

Guarantor The scientific guarantor of this publication is Simon Fandler-Höfler.

Conflict of interest The authors of this manuscript declare no conflicts of interest related to the subject matter of the article.

Statistics and biometry No complex statistical methods were necessary for this Editorial Comment.

Informed consent Written informed consent was not required for this study because it is an Editorial Comment.
Ethical approval Ethical approval was not required for this study because it is an Editorial Comment.

Methodology

- Editorial Comment

\section{References}

1. Silvis SM, de Sousa DA, Ferro JM, Coutinho JM (2017) Cerebral venous thrombosis. Nat Rev Neurol 13:555-565. https://doi.org/ 10.1038/nrneurol.2017.104

2. Ferro JM, Canhão P, Stam J et al (2009) Delay in the diagnosis of cerebral vein and dural sinus thrombosis: influence on outcome. Stroke 40:3133-3138. https://doi.org/10.1161/STROKEAHA.109. 553891

3. Buyck P-J, Zuurbier SM, Garcia-Esperon C et al (2019) Diagnostic accuracy of noncontrast CT imaging markers in cerebral venous thrombosis. Neurology 92:e841-e851. https://doi.org/10. 1212/WNL.0000000000006959

4. van Dam LF, van Walderveen MAA, Kroft LJM et al (2020) Current imaging modalities for diagnosing cerebral vein thrombosis - a critical review. Thromb Res 189:132-139. https://doi.org/10. 1016/j.thromres.2020.03.011

5. Xu W, Gao L, Li T, Ramdoyal ND, Zhang J, Shao A (2018) The performance of CT versus MRI in the differential diagnosis of cerebral venous thrombosis. Thromb Haemost 118:1067-1077. https://doi.org/10.1055/s-0038-1642636

6. Yang Q, Duan J, Fan Z et al (2016) Early detection and quantification of cerebral venous thrombosis by magnetic resonance blackblood thrombus imaging. Stroke 47:404-409. https://doi.org/10. 1161/STROKEAHA.115.011369

7. Niu P-P, Yu Y, Guo Z-N et al (2016) Diagnosis of non-acute cerebral venous thrombosis with 3D T1-weighted black blood sequence at 3T. J Neurol Sci 367:46-50. https://doi.org/10.1016/j. jns.2016.05.052

8. Yang X, Wu F, Liu Y et al (2021) Diagnostic performance of MR black-blood thrombus imaging for cerebral venous thrombosis in real-world clinical practice. Eur Radiol. https://doi.org/10.1007/ s00330-021-08286-x

Publisher's note Springer Nature remains neutral with regard to jurisdictional claims in published maps and institutional affiliations. 